Results Studies carried out in 4 continents and 14 countries while recruiting female and male players aging range from 9 to 30 . These indicate that the $11+$ has been investigated worldwide. Quality of studies was moderate to high and except an unclear amount of bias for blinding outcome assessment; risk of bias for all domains was low. Excepting lower extremity stability, ankle evertors time latency and proprioception improvement, application of the $11+$ at long-term (a complete football season) appeared to be successful in improving a variety of performance tests e.g. agility, sprinting, balance, jumping, cutting maneuvers etc; physiological responses and a majority of biomechanical measures. Vice versa, that the $11+$ causes acute negative impact on the physical performance and technical abilities compared to the dynamic warm ups was highlighted in two studies.

Conclusion Application of the $11+$ as warm up routine during trainings at long-term with higher adherence can be recommended for improving performance. However, caution must be observed while recruiting the $11+$ for warming up before competitions as it may acutely decrease physical performance and technical abilities. Given the contradictory nature of the literature, further studies should evaluate short-term effects of the programme.

\section{WHAT ABOUT BMX? A SCOPING REVIEW OF INJURIES, RISK FACTORS, AND PREVENTION STRATEGIES}

1,2,3,4Amanda M Black, 'Srijal Gupta, ' Claire Rockcliff. 'Sport Injury Prevention Research Centre, Faculty of Kinesiology, University of Calgary, Calgary, Canada; ${ }^{2}$ Alberta Children's Hospital Research Institute, University of Calgary, Calgary, Canada; ${ }^{3} \mathrm{O}^{\prime} B$ rien Institute for Public Health, University of Calgary, Calgary, Canada; ${ }^{4}$ Hotchkiss Brain Institute, University of Calgary, Calgary, Canada

\subsection{6/bjsports-2021-IOC.191}

Background Bicycle motocross (BMX) was officially added to the Olympics in 2008. Participation has increased over the last decade and is listed as a top sport for injury rates in multisport studies. Before effective prevention programs can be designed and implemented, it is important to understand injury risk, risk factors and potential prevention strategies.

Objective To examine the evidence on injury incidence, prevalence, risk factors, prevention strategies, and prevention implementation in BMX.

Methods Five electronic databases were systematically searched in July 2020 for studies that included BMX injury as the main topic or subtopic. Two reviewers screened all studies and extracted data independently. Conflicts were resolved via consensus and a third reviewer.

Results Of the 1615 unique articles screened, 36 met the inclusion criteria. Most injury surveillance based studies were conducted at elite competitions (e.g. BMX Cycling European Championship, Olympic Games, UCI BMX World Championship) or using data from the emergency department. The most common BMX injuries were fractures, lacerations, abrasions, and contusions. Risk factors included age, sex, number of riders per race, history of injury, and bicycle characteristics. Prevention strategies are limited and have not been appropriately evaluated; one study found that wearing a neck brace may reduce the number and magnitude of rotational accelerations at the head during BMX racing, but this was not evaluated for its effect on injury rates.
Conclusions Most BMX studies focus on injury characteristics and do not use appropriate injury surveillance methodology. Studies based on emergency room data may underestimate less severe injuries and do not provide adequate measures of sport exposure. Reducing the number of riders per race may be a promising modifiable risk factor that requires further examination. More rigorous community-based prospective studies examining injury rates, risk factors, and prevention strategies are needed to inform widespread evidence-based prevention strategies.

\section{PERFORMANCE OF INERTIAL MEASUREMENT UNITS TO CLASSIFY DIFFERENT UPPER LIMB ACTIVITIES IN ELITE WOMEN'S WATER POLO: A CROSS SECTIONAL STUDY}

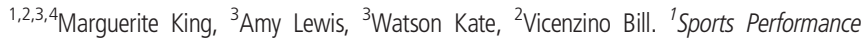
Innovation and Knowledge Excellence (SPIKE), Queensland Academy of Sport, Brisbane, Australia; ${ }^{2}$ School of Health and Rehabilitation Sciences, University of Queensland, Brisbane, Australia; ${ }^{3}$ Queensland Academy of Sport, Brisbane, Australia; ${ }^{4}$ Queensland Sports Medicine Centre, Brisbane, Australia

\subsection{6/bjsports-2021-IOC.192}

Background Water polo had the highest injury incidence of all team sports at the 2016 Rio Olympics. Injuries have been linked to external load in training and competition (Mountjoy et al, 2015, Wheeler et al, 2013). External load monitoring is likely instructive in managing loads to optimise performance while minimising injury risk. Inertial measurement units and machine learning techniques have shown promise in other sports in monitory external load.

Objective To investigate the performance of a decision tree model using peak resultant acceleration (RAcc $\left.{ }^{\mathrm{PEAK}}\right)$ and angular velocity (AngVel ${ }^{\text {PEAK}}$ ) variables during water polo activities high intensity throwing (HIT), low intensity throwing (LIT), blocking with ball contact (BWBC) and swimming (SWIM) compared to video analysis.

Design A cross-sectional study

Setting A sports institute pool.

Participants We recruited ten elite female water polo players 21.2 (SD 4.8) years old, 8.7 (SD 4.6) training years.

Interventions Two Blue Trident ${ }^{\mathrm{TM}}$ inertial measurement units were applied to each athlete to collect kinetic variables during a standardised baseline test. Each test was recorded using a digital video recorder and coded for activity verification. RStudio was used to analyse the data and calculate the predicted volume of each activity compared to the coded video verification.

Main Outcome Measurements RAcc ${ }^{\text {PEAK }}$ and AngVel ${ }^{\text {PEAK }}$ values in abovementioned activities and model accuracy.

Results Each of the activities showed distinct bandwidths of

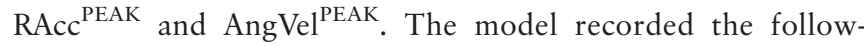
ing activity volume $(\mathrm{N})$ with 6 false positive errors with a 96\% accuracy - HIT (32) LIT (34), BWBC (22) and SWIM (70).

Conclusions This method shows potential to identify different upper limb activities in water polo. These activities most likely load the upper limb differentially and would be critical to measure in monitoring external training load. External load measurement may assist in optimising training planning. 